\title{
New designing for nanostructured 2D materials and 2D superlattices
}

\author{
Yao Xiao and Lei $\mathrm{Fu}^{1,2^{*}}$
}

As the most remarkable candidate for the next-generation electronics, two-dimensional (2D) materials have attracted much interest. Recently in a letter in Nature Materials, Professor David A. Muller and Lain-Jong Li and their cooperators reported the direct synthesis of one-dimensional (1D) $\mathrm{MoS}_{2}$ channels embedded in 2D $\mathrm{WSe}_{2}$ monolayers through a dislocation-catalyzed strategy [1]. They demonstrated that $1 \mathrm{D} \mathrm{MoS}$ channels of subnanometer widths were formed in the second-step of a two-step chemical vapor deposition (CVD) growth of the lateral $\mathrm{WSe}_{2} / \mathrm{MoS}_{2}$ heterostructures. The root cause lies in the exposure of the as-generated $5 \mid 7$ member ring dislocations along the sharp grain boundaries or implanted in $\mathrm{WSe}_{2}$ to the Mo and $\mathrm{S}$ atmosphere. Through atomic resolution annular dark-field scanning transmission electron microscopy (ADF-STEM) and molecular dynamics (MD) simulations, a conclusion was drawn that the higher reactivity in the core of the misfit dislocations induced the insertion of Mo and S atoms and such dislocations were pushed away from the ordinary disloca- tions to form 1D channels (Fig. 1). Moreover, a 1D $\mathrm{MoS}_{2}$ nanowire of $\sim 1 \mathrm{~nm}$ long could be obtained from the periodic dislocation along the boundary of two $\mathrm{WSe}_{2}$ grains. The work could provide with the inspiration of the synthesis of 1D channels based on the substrate of defects. That is, different channels based on 2D materials could be fabricated through chemical methods which prevent from the impurities caused by lithography. It is essential for a device to simplify the constructing process in order to reduce the introduction of impurities. According to this work, the substrate design is critical to the material fabrication.

Till now, plenty of substrate-designing strategies have been generally utilized to fabricate nanostructured $2 \mathrm{D}$ materials or superlattices. Here, the substrates refer not only to metals, $\mathrm{SiO}_{2} / \mathrm{Si}$, mica, sapphire, but also to edges, grains or even defects of 2D materials. Thus, CVD growth for the synthesis of nanostructured 2D materials and superlattices lies most on the substrate designing. There have been a series of methods to design metal or non-

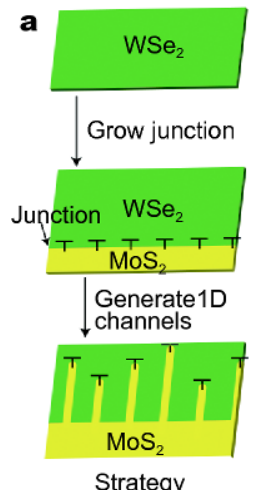

Strategy
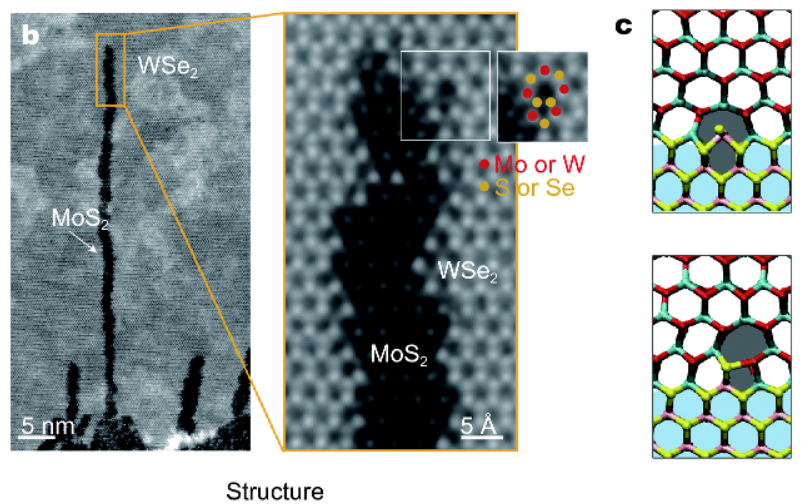

Structure

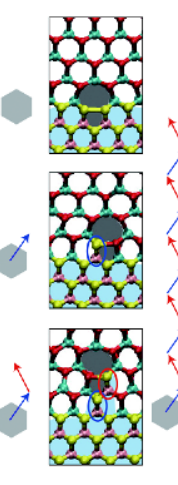

Mechanism

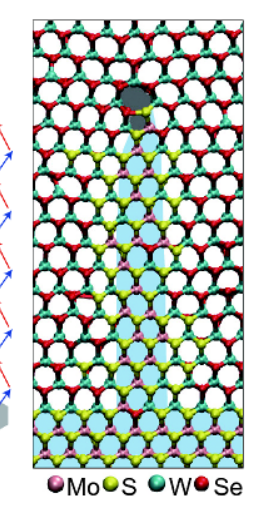

Figure 1 Schemes illustrating the strategy, structures and mechanism of the construction of $1 \mathrm{D}$ MoS 2 channels. Adapted with permission from Ref. [1] (Copyright 2017, Nature Publishing Group).

\footnotetext{
${ }^{1}$ The Institute for Advanced Studies (IAS), Wuhan University, Wuhan 430072, China

${ }^{2}$ College of Chemistry and Molecular Sciences, Wuhan University, Wuhan 430072, China

*Email: leifu@whu.edu.cn
} 
metal substrates. For instance, Liu et al. reported the fabrication of the graphene nanoribbon (GNR) array via substrate wrinkle engineering [2], and Fu et al. demonstrated the twinned growth of $\mathrm{ReS}_{2} / \mathrm{WS}_{2}$ heterostructures through a liquid metal CVD process [3]. Such strategies could change the morphology or component of the substrates, directly impacting the grown products. The epitaxy growth along the edges or from the grains is related to the lattice mismatch, such as the lateral $1 \mathrm{~T}^{\prime}-\mathrm{MoTe}_{2} / 1 \mathrm{H}-$ $\mathrm{MoS}_{2}, 1 \mathrm{~T}^{\prime}-\mathrm{MoTe}_{2} / 2 \mathrm{H}-\mathrm{MoTe}_{2}$ superlattices and the multinary lateral superlattices of $\mathrm{MoS}_{2}, \mathrm{WS}_{2}$ and $\mathrm{WSe}_{2}$ [4-6]. Even so, such a dislocation driven growth is so general that it is possible to obtain other kinds of nanowires or superlattices on the basis of defects in 2D materials. That is, not only misfit dislocation but also atomic vacancy or unexpected doping could serve as "substrates", which would open up a new field of fabricating strategies.

Nanostructured 2D materials are also attractive, especially for their nanowires or nanoribbons. Due to the high reactivity of the edge atoms, isolated nanowires or nanoribbons of most $2 \mathrm{D}$ materials would be unstable except graphene and hexagonal boron nitride (h-BN). It is very difficult to directly obtain $1 \mathrm{D}$ channels such as $1 \mathrm{D}$ transition metal dichalcogenides (TMDs) though thin channels could sustain the large strain and have access to a wider range of electronic bands [1]. Up to now, lithographic patterning is a widely used strategy to construct 2D heterostructure devices. Unfortunately, the consequent atomic defects and contamination affect the performance of the devices due to the electronic defect states. Therefore, inspired by such dislocation-catalyzed growth, other innovative methods could be developed for other $1 \mathrm{D}$ channels or $1 \mathrm{D}-2 \mathrm{D}$ superlattices. These nanostructured 2D materials or superlattices would be compatible with high-frequency electronics, such as narrow channel field-effect transistors (FETs) or vertical FETs (VFETs), which are important for high-speed and lowpower devices $[7,8]$.

Theoretical computations need further development. Except for graphene and h-BN, the mechanism of other 2D materials' growth still remains unclear. Herein, this work provides a good example, in which the density functional theory (DFT) and MD calculations are used to explain how the $1 \mathrm{D} \mathrm{MoS}$ channel is formed by the misfit dislocation. Theoretical researches of $2 \mathrm{D}$ materials growth could be a significant guidance for the structure design, catalyst selection and superstructure engineering.

Received 18 December 2017; accepted 19 December 2017; published online 27 December 2017

1 Han Y, Li MY, Jung GS, et al. Sub-nanometre channels embedded in two-dimensional materials. Nat Mater, doi: 10.1038/nmat5038

2 Pan Z, Liu N, Fu L, et al. Wrinkle engineering: a new approach to massive graphene nanoribbon arrays. J Am Chem Soc, 2011, 133: 17578-17581

3 Zhang T, Jiang B, Xu Z, et al. Twinned growth behaviour of twodimensional materials. Nat Commun, 2016, 7: 13911

4 Naylor CH, Parkin WM, Gao Z, et al. Synthesis and physical properties of phase-engineered transition metal dichalcogenide monolayer heterostructures. ACS Nano, 2017, 11: 8619-8627

5 Sung JH, Heo H, Si S, et al. Coplanar semiconductor-metal circuitry defined on few-layer $\mathrm{MoTe}_{2}$ via polymorphic heteroepitaxy. Nat Nanotechnol, 2017, 12: 1064-1070

6 Zhang Z, Chen P, Duan X, et al. Robust epitaxial growth of twodimensional heterostructures, multiheterostructures, and superlattices. Science, 2017, 357: 788-792

$7 \mathrm{Yu}$ WJ, Li Z, Zhou H, et al. Vertically stacked multi-heterostructures of layered materials for logic transistors and complementary inverters. Nat Mater, 2012, 12: 246-252

8 Cheng R, Jiang S, Chen Y, et al. Few-layer molybdenum disulfide transistors and circuits for high-speed flexible electronics. Nat Commun, 2014, 5: 5143 